\title{
Impact of maternal probiotic-supplemented dietary counselling on pregnancy outcome and prenatal and postnatal growth: a double-blind, placebo-controlled study
}

\author{
Raakel Luoto $^{1,2} *$, Kirsi Laitinen ${ }^{3,4}$, Merja Nermes ${ }^{1}$ and Erika Isolauri ${ }^{1,2}$ \\ ${ }^{1}$ Department of Paediatrics, Turku University Hospital, Kiinamyllynkatu 4-8, 20520 Turku, Finland \\ ${ }^{2}$ Department of Clinical Sciences, University of Turku, Turku, Finland \\ ${ }^{3}$ Department of Biochemistry and Food Chemistry, University of Turku, 20014 Turku, Finland \\ ${ }^{4}$ Functional Foods Forum, University of Turku, 20014 Turku, Finland \\ (Received 18 August 2009 - Revised 25 November 2009 - Accepted 18 December 2009 - First published online 4 February 2010)
}

\begin{abstract}
The perinatal nutritional environment impacts upon the health and well-being of mother and child also in the long term. The aim of the present study was to determine the safety and efficacy of perinatal probiotic-supplemented dietary counselling by evaluating pregnancy outcome and fetal and infant growth during the 24 months' follow-up. Altogether, 256 women were randomised at their first trimester of pregnancy into a control and a dietary intervention group. The intervention group received intensive dietary counselling provided by a nutritionist and were further randomised, double-blind to receive probiotics (Lactobacillus rhamnosus GG and Bifidobacterium lactis Bb12; diet/probiotics) or placebo (diet/placebo). Firstly, probiotic intervention reduced the frequency of gestational diabetes mellitus (GDM); $13 \%$ (diet/probiotics) v. 36\% (diet/placebo) and $34 \%$ (control); $P=0.003$. Secondly, the safety of this approach was attested by normal duration of pregnancies with no adverse events in mothers or children. No significant differences in prenatal or postnatal growth rates among the study groups were detected. Thirdly, distinctive effects of the two interventions were detected; probiotic intervention reduced the risk of GDM and dietary intervention diminished the risk of larger birth size in affected cases; $P=0.035$ for birth weight and $P=0.028$ for birth length. The results of the present study show that probiotic-supplemented perinatal dietary counselling could be a safe and cost-effective tool in addressing the metabolic epidemic. In view of the fact that birth size is a risk marker for later obesity, the present results are of significance for public health in demonstrating that this risk is modifiable.
\end{abstract}

Diabetes: Gestational diabetes mellitus: Dietary counselling: Pregnancy: Probiotics

The fetal and neonatal periods critically guide the development, growth and functional maturation of the organ systems. There are data to suggest that small birth weight with rapid postnatal catch-up growth on the one hand or large birth weight on the other hand is associated with major disorders in adult life, including CVD, hypertension, non-insulindependent diabetes and obesity ${ }^{(1-3)}$. Maternal nutrition constitutes a decisive factor in the in utero environment. Infants of small birth weight reflecting poor intra-uterine nutrition have a heightened risk of such chronic diseases, a phenomenon called programming ${ }^{(4)}$. In Western societies, in contrast, the fetus is often overnourished due to the mother's excessive dietary intake of saturated fat and carbohydrates of high glycaemic index. Maternal nutrition also influences the composition of breast milk, with a marked growth-promoting and regulatory potential against infections and allergies ${ }^{(5,6)}$. Additionally, maternal prepregnancy weight correlates with the birth weight of the child, and maternal obesity is linked to fetal macrosomia ${ }^{(7)}$, these being associated per se with later obesity ${ }^{(2,3)}$. Maternal obesity is also a well-known contributor of gestational diabetes mellitus (GDM), which elevates itself the risk of non-insulin-dependent diabetes and the metabolic syndrome later in life ${ }^{(8)}$. We therefore hypothesised here that the nutritional environment during the perinatal period creates a window of opportunity to reverse the increasing trend in Western lifestyle diseases.

Recent data also point to the gut microbiota as being instrumental in the microbial, metabolic and immunological programming of the child ${ }^{(9,10)}$. Indeed, aberrant compositional development of the gut microbiota has been linked to obesity $^{(11,12)}$ and an allergy risk ${ }^{(13)}$, and modification of the gut microbiota by probiotics early in life has thus attracted scientific interest. However, the long-term safety of probiotic intervention during the period when the bases of child's immunological and metabolic phenotypes are created remains to be established.

The objective of our Nutrition, Allergy, Mucosal immunology and Intestinal microbiota programme is to accomplish clinical intervention studies with perinatal probiotic intervention combined with balanced maternal nutrition during

Abbreviation: GDM, gestational diabetes mellitus.

* Corresponding author: Raakel Luoto, fax +358 2313 1460, email raakel.luoto@utu.fi 
pregnancy and lactation and to provide a new direction in the search for scientifically validated means of reducing the risk of Western lifestyle disease. In our recent publications, we have provided evidence of perinatal dietary counselling to improve the dietary intake of the pregnant women ${ }^{(14)}$ and perinatal probiotic intervention to contribute to improved glucose regulation during and after pregnancy ${ }^{(15)}$. The present study specifically evaluates the safety of this approach. To this end, a mother-infant cohort ( $n$ 256) was followed with careful evaluation of pregnancy outcome and health and growth of the children.

\section{Subjects and methods}

\section{Study design}

The original study population comprised 256 mother-baby pairs participating in an ongoing prospective, randomised mother-infant nutrition and probiotic study (http://www. clinicaltrials.gov/ct/gui/show/NCT00167700,section3), described in detail elsewhere ${ }^{(14-16)}$. In brief, women were recruited in early pregnancy during their first visit to maternal welfare clinics in Turku and neighbouring areas in South-West Finland. The inclusion criterion was that the subjects had no chronic metabolic diseases; however, the allergic diseases (atopic eczema, allergic rhinitis or asthma) were allowed. At entry, the women were randomised into a dietary intervention group or a control group. All women received dietary counselling provided by welfare clinics according to a national programme. The intervention group received additionally intensive dietary counselling at every study visit provided by a nutritionist, the aim being a dietary intake complying with current recommendations ${ }^{(17,18)}$, combined with conventional food products with favourable fat and fibre contents for use at home. This group was further randomised at baseline in a double-blind manner to receive either probiotics (diet/ probiotics group), Lactobacillus rhamnosus GG (ATCC 53 103, Valio Ltd, Helsinki, Finland) and Bifidobacterium lactis Bb12 (Chr. Hansen, Hørsholm, Denmark) at a dose of $10^{10}$ colony-forming units/d each, or placebo (diet/placebo group), microcrystalline cellulose and dextrose anhydrate (Chr. Hansen) capsules. The control group received placebo capsules in a single-blinded manner (Fig. 1). The capsules were taken once daily and the intervention period extended from the first trimester of pregnancy to the end of exclusive breastfeeding. The present study was conducted according to the guidelines laid down in the Declaration of Helsinki, and all procedures involving human participants were approved by the Ethical Committee of the Hospital District of Southwest Finland. Written informed consent was obtained from all participants.

As depicted in Fig. 1, altogether, 256 pregnant women were recruited and 238 of them continued the study throughout pregnancy. Three twin pairs were excluded from the growth follow-up. Of the 241 children delivered, 191 completed the 24 months' follow-up.

\section{Clinical evaluation}

Pregnant women. The women in all study groups visited the study clinic three times during pregnancy, in addition to their regular visits to well-women clinics. Their clinical data were collected by interview at the first visit (Table 1). The duration of pregnancy was calculated from the date of the last menstruation. The results of $75 \mathrm{~g}$ oral glucose tolerance tests were recorded; these were performed in welfare clinics in all risk pregnancies. The diagnosis of GDM was based on modified criteria of the Fourth International WorkshopConference on Gestational Diabetes Mellitus ${ }^{(19)}$, according to recommendations implemented in Finland during the clinical study years 2002-2005. Specifically, an oral glucose tolerance test was considered pathological when one value exceeded $\geq 4.8 \mathrm{mmol} / \mathrm{l}$ at baseline, $\geq 10.0 \mathrm{mmol} / \mathrm{l}$ at $1 \mathrm{~h}$ or $\geq 8.7 \mathrm{mmol} / \mathrm{l}$ at $2 \mathrm{~h}$. The estimate of fetal weight in the $20 \mathrm{th}$ pregnancy week ultrasound examination was recorded in cases where the women were willing to have the test performed. These examinations, provided by the mother's municipality, were performed by sonographers or doctors experienced in obstetric ultrasound. The duration of exclusive and total breastfeeding was recorded from the mother.

Infant. After delivery, study visits were scheduled at the ages of 1, 6, 12 and 24 months. Infant weight was measured with a SECA model 727 scale (Hamburg, Germany) and length on a length board. All weights and lengths were recorded to the nearest $0.01 \mathrm{~kg}$ and $0.1 \mathrm{~cm}$, respectively. Physical examinations were always carried out by the same research nurse at the age of 1 month and by a physician in the research group at 6,12 and 24 months of age, apart from the regular visits to the well-baby clinics. The clinical evaluation of the infants included inspections of skin, ears, eyes, nose, genitalia and anus, auscultation of heart and lungs, palpation of abdomen and femoral pulsations, evaluation of growth and nutritional status, muscle tone and neurological development.

\section{Statistical methods}

Pregnancy outcome and infant growth were the primary outcome variables. The baseline variables and other variables in Table 1 were analysed using the $\chi^{2}$ test, ANOVA and Kruskal-Wallis test (5-min Apgar score). Perinatal death was defined as stillbirth at 23 weeks or more of gestation or death of an infant less than 1 week of age. Very premature delivery was defined as spontaneous live birth at weeks 23-31, premature delivery as spontaneous live birth at weeks $32-36$, term delivery as spontaneous live birth at weeks $37-41$ and postterm delivery as spontaneous live birth $\geq 42$ weeks of gestation.

All growth measurements were estimated to precise daily ages (1, 6, 12 and 24 months) using linear interpolating by reason of variable measuring ages $(1,0.6$ and 2.16 months; $6,5.0$ and 7.7 months; $12,10.8$ and 16.7 months; $24,22.3$ and 33.6 months). Missing data for length (n 12) and weight ( $n$ 8) were linearly interpolated using the exact daily ages; no extrapolation was made. Growth rate was defined as grams and centimetres gained per month between the two measurements. Comparison of growth rates during the periods 0-6 months, 6-12 months and 12-24 months among the study groups was made by ANOVA for repeated measurements.

As confounders, we considered mothers' BMI before pregnancy $\left(\mathrm{kg} / \mathrm{m}^{2}\right)$, maternal smoking before pregnancy (yes or no), highest maternal education in terms of college or university (yes or no), GDM, sex and breastfeeding at infant's age of 


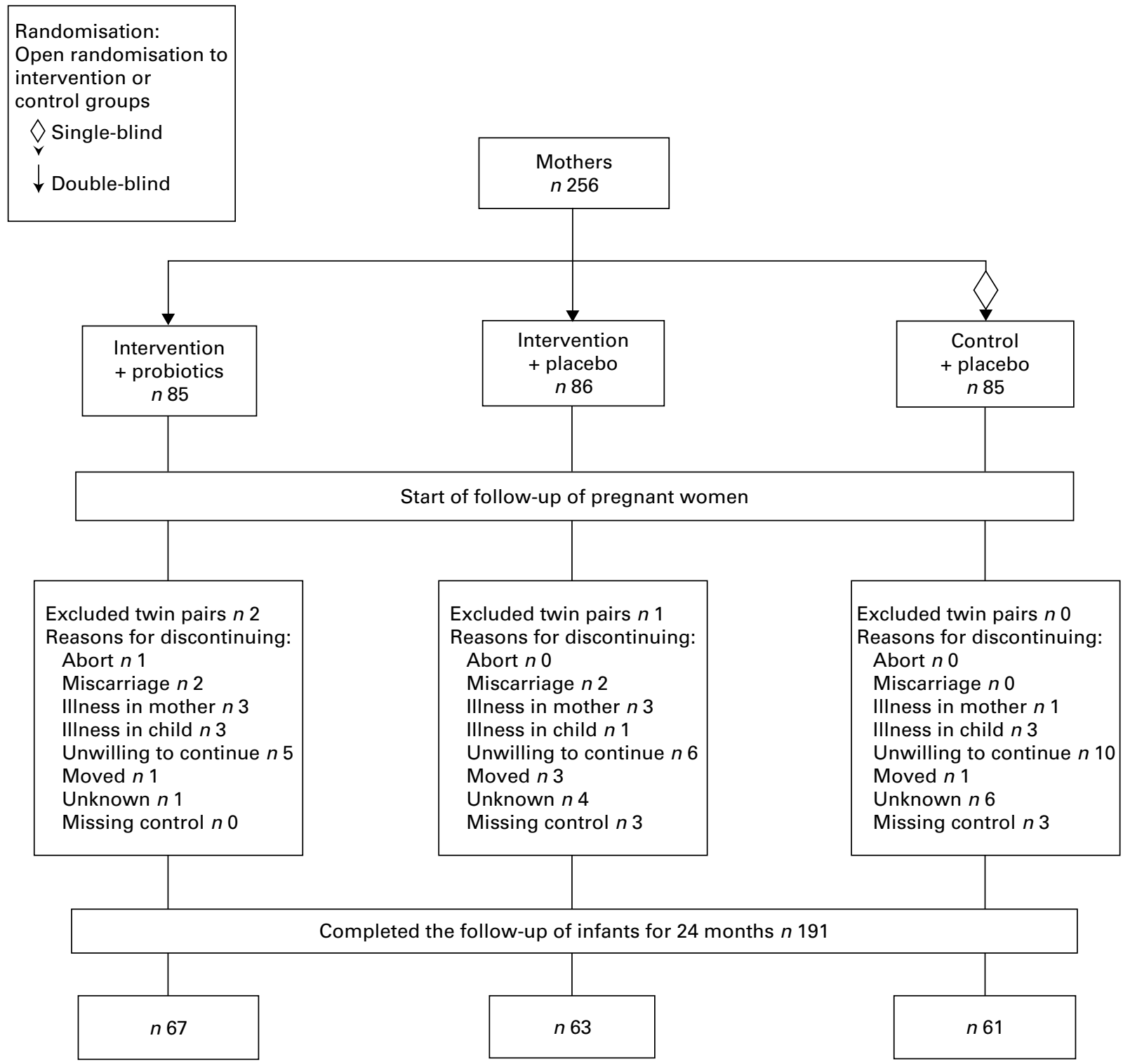

Fig. 1. The flow of subjects.

6 months (yes or no). Associations between confounders and infant growth rates at separate time periods were studied by including those confounders as continuous or dichotomous covariates in multivariate models. Logistic regression analysis was used to compare the incidence of GDM among the study groups. The results are given as OR with $95 \%$ CI. Two-way ANOVA with two explaining factors, study group and GDM, was used to analyse the birth size variables. Due to significant interactions between dietary intervention and GDM, subgroup analyses were conducted to estimate the effect of GDM in study groups.

Data were analysed using SPSS version 15.0 (SPSS Inc., Chicago, IL, USA). A $P$ value of $<0.05$ was considered statistically significant.

\section{Results}

As shown in Table 1, the mean age of the women was $30 \cdot 0$ (range 17.6-44.2) years. All participants were Caucasian and healthy; 201 (79\%) had previously been diagnosed with an allergic disease. The mean BMI of women was 23.6 (SD 3.8$) \mathrm{kg} / \mathrm{m}^{2(16)}$. The majority had college or university education and were primipara.

\section{Pregnancy outcome}

Pregnancy outcome is depicted in Table 1. The frequency of GDM was significantly different between study groups $(P=0.003$, Table 1$)$. The risk of GDM was significantly reduced in the diet/probiotics group when compared to the control group $(\mathrm{OR}=0.27$ (95\% CI 0.11, 0.62); $P=0.002)$, whereas in the diet/placebo group, the risk was not significantly different when compared to the control group $(\mathrm{OR}=1.08(95 \%$ CI $0.55,2.12) ; P=0.823)$. There were no significant differences among the study groups in the duration of gestation. There were altogether 4/238 (1.7\%) preterm deliveries, only one of them, a twin pregnancy, coming very preterm (30th gestation week). Post-term deliveries were 
Table 1. Clinical characteristics of the women and impact of the intervention on pregnancy outcome

\begin{tabular}{|c|c|c|c|}
\hline Group... & Diet/probiotics ( $n$ 85) & Diet/placebo ( $n$ 86) & Control $(n 85)$ \\
\hline \multicolumn{4}{|c|}{ Clinical characteristics of mothers and pregnancies } \\
\hline Age (years) & $29 \cdot 7(4 \cdot 1)$ & $30 \cdot 1(5 \cdot 2)$ & $30.2(5.0)$ \\
\hline Higher degree education & $68(80 \%)$ & $59(69 \%)$ & $67(79 \%)$ \\
\hline Primipara & $55(65 \%)$ & $44(51 \%)$ & $48(56 \%)$ \\
\hline Twin pregnancies & $2(2 \%)$ & $1(1 \%)$ & $0(0 \%)$ \\
\hline \multicolumn{4}{|l|}{ Impact on pregnancies } \\
\hline Miscarriages $<22$ weeks & $2(2 \%)$ & $2(2 \%)$ & $0(0 \%)$ \\
\hline Gestational diabetes mellitus* & $10 / 76(13 \%)$ & $27 / 76(36 \%)$ & $25 / 73(34 \%)$ \\
\hline Duration of gestation (weeks) $\dagger$ & $40 \cdot 0(36 \cdot 9,42 \cdot 8)$ & $40.0(30.5,42.4)$ & $40.0(34.9,43.3)$ \\
\hline$<32$ & $0 / 80(0 \%)$ & $1 / 79(1 \%)$ & $0 / 79(0 \%)$ \\
\hline $32-36$ & $2 / 80(2 \%)$ & $0 / 79(0 \%)$ & $1 / 79(1 \%)$ \\
\hline $37-41$ & $73 / 80(91 \%)$ & $72 / 79(91 \%)$ & $74 / 79(94 \%)$ \\
\hline$=42$ & $6 / 80(7 \%)$ & $6 / 79(8 \%)$ & $4 / 79(5 \%)$ \\
\hline Caesarean delivery & $12 / 75(16 \%)$ & $12 / 77(16 \%)$ & $11 / 76(14 \%)$ \\
\hline Impact on the neonates & $(n 82)$ & $(n 80)$ & $(n 79)$ \\
\hline Sex (male) & $43(52 \%)$ & $40(50 \%)$ & $45(57 \%)$ \\
\hline 5-min Apgar scoret & $9(6-10)$ & $9(3-10)$ & $9(4-10)$ \\
\hline Birth weight $(\mathrm{g})$ & $3467(3370,3564)$ & $3579(3469,3688)$ & $3611(3494,3727)$ \\
\hline Birth length $(\mathrm{cm})$ & $50 \cdot 7(50 \cdot 3,51 \cdot 1)$ & $51.2(50.7,51.6)$ & $51.0(50.5,51.5)$ \\
\hline Head circumference at birth $(\mathrm{cm})$ & $34.8(34.5,35.1)$ & $35.0(34.7,35.3)$ & $35.2(34.9,35.5)$ \\
\hline
\end{tabular}

Results are given as mean (SD), mean $(95 \% \mathrm{Cl})$ or as numbers $(\%)$ of subjects, if not stated otherwise. Total number of subjects is given if all are not included.

${ }^{*}$ None of the differences among the groups was significant except $P=0.003$.

† Median (range).

more common, in 16/238 (7\%) pregnancies, with no significant differences in frequency among the study groups.

Rates of caesarean delivery were $14-16 \%$ in all study groups. There were no perinatal deaths or serious adverse incidences such as sepsis in mothers or newborns. The 5-min Apgar scores were inline in all study groups (Table 1). Likewise, the mean duration of exclusive breastfeeding and thus the duration of probiotic/placebo intervention did not differ among the study groups; 2.9 (SD 1.7) months in the diet/probiotics group, 3.4 (SD 1.8) months in the diet/placebo group and 3.0 (SD 1.6) months in the control group. Neither did the groups diverge significantly with regard to breastfeeding status at 6 months of age; altogether, $68 \%$ in the diet/probiotics group, $75 \%$ in the diet/placebo group and $76 \%$ in the control group were breastfed to any degree at least for 6 months.

\section{Growth}

The mean birth weight of the neonates ( $n$ 241) was 3551 (range 1610-4750) $\mathrm{g}$ and birth length 50.9 (range 44.0$57.0) \mathrm{cm}$, showing no significant differences among the study groups (Table 1). Moreover, when the birth weights and lengths were adjusted to mothers' prepregnancy BMI $\left(\mathrm{kg} / \mathrm{m}^{2}\right)$, smoking before pregnancy, education, GDM, sex of the child and duration of breastfeeding, no statistically significant differences emerged among the study groups. GDM tended to increase birth weight and birth length in all study groups without a significant main effect $(P=0.183$ and $0 \cdot 278$, respectively, two-way ANOVA; Table 2). In spite of non-significant main effects, the interactions $(P=0.054$ for birth weight and 0.034 for birth length) and the following subgroup analyses indicated that the impact of GDM on birth size was manifested most distinctly in the control group $(426 \mathrm{~g}$ in weight and $1.7 \mathrm{~cm}$ in length; $P=0.001$ ), and the effect of GDM on birth size was lessened to non-significant effects of 190 and $73 \mathrm{~g}$ in weight and 0.6 and $0.1 \mathrm{~cm}$ in length by dietary intervention with or without probiotics (Table 2). The effect of dietary intervention and GDM on birth size was analysed further and the dietary intervention groups were combined (Table 3). Again, interactions were detected between dietary intervention and GDM $(P=0.035$ for birth weight and 0.028 for birth length), indicating that the dietary intervention modified the effect of GDM. GDM increased the birth weight on the average $144(95 \% \mathrm{CI}-7,295) \mathrm{g}$ and $0.4(95 \% \mathrm{CI}-0.2,1.1) \mathrm{cm}$ in the combined dietary intervention group and 426 (95\% CI 193, 660) g and $1.7(95 \% \mathrm{CI}$ $0 \cdot 7,2 \cdot 7) \mathrm{cm}$ in the control group.

Weight gain and growth in length during the periods $0-6$ months, 6-12 months and 12-24 months showed no statistically significant differences between the diet/probiotics, diet/ placebo and control groups. The respective mean weight gains (g/month) over 0-6 months were 759 (SD 160), 762 (SD 165) and 756 (SD 148); over 6-12 months were 323 (SD 80), 296 (SD 99) and 315 (SD 91); over 12-24 months were 211 (SD 76), 230 (SD 61) and 218 (SD 52) $(P=0.983$ for group effect; $P<0.001$ for time effect; $P=0.520$ for group $\times$ time interaction; ANOVA for repeated measurements). The mean growths in length $(\mathrm{cm} /$ month) in the respective periods were 2.84 (SD 0.35), 2.89 (SD 0.29) and 2.93 (SD 0.35); 1.40 (SD 0.19), 1.38 (SD 0.21 ) and 1.36 (SD 0.24); 0.95 (SD 0.14), 0.94 (SD 0.15) and 0.93 (SD 0.12) in the diet/probiotics, diet/placebo and control groups, respectively $(P=0.872$ for group effect; $P<0.001$ for time effect; $P=0.325$ for group $\times$ time interaction; ANOVA for repeated measurements).

In the subgroup of infants undergoing the 20th pregnancy week ultrasound examination (diet/probiotics group $n 28$, diet/placebo group $n 25$ and control group $n$ 17), there were no statistically significant differences among the study groups in perinatal weight gain or weight gain and growth in length over the 24 months' follow-up $(P=0 \cdot 809$, ANOVA for repeated measurements, group effect; Fig. 2). 


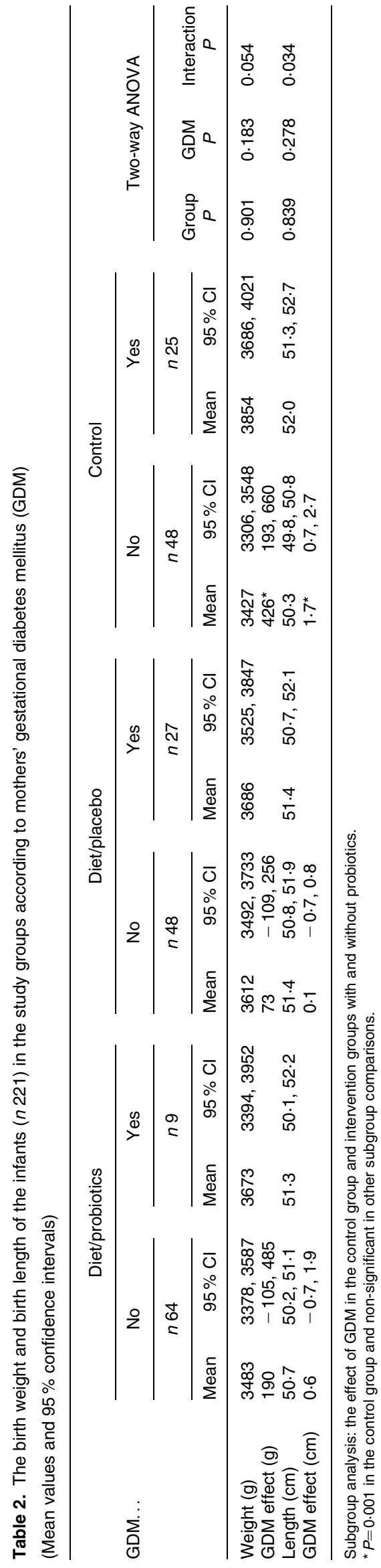

As an additional analysis, the adjusted group comparisons for periods of $0-6,6-12$ and $12-24$ months were performed separately in order to evaluate the effect of other confounders. In the 0-6 months' age period, the adjusted group comparisons to explain weight gain and growth in length were non-significant $(P=0.905$ and 0.441 , respectively). However, male sex increased the weight gain by $94 \mathrm{~g} / \mathrm{month}$ $(P<0.001)$, and breastfeeding for at least 6 months decreased the weight gain by $70 \mathrm{~g} /$ month $(P=0 \cdot 003)$. Likewise, male sex increased growth in length during the $0-6$ months' age period by $0.23 \mathrm{~cm} /$ month $(P<0.001)$, and breastfeeding for at least 6 months decreased growth in length by $0.15 \mathrm{~cm} / \mathrm{month}$ $(P=0.002)$. The adjusted group comparisons with respect to weight and length gain were also non-significant during the periods 6-12 months and 12-24 months of age. Breastfeeding for at least 6 months decreased statistically significantly the growth in length during the 6-12 months' age period by $0.07 \mathrm{~cm} /$ month $(P=0.028)$. Male sex decreased the growth in length $0.05 \mathrm{~cm} /$ month $(P=0.144)$ during $6-12$ months' and $0.04 \mathrm{~cm} /$ month $(P=0.029)$ during $12-24$ months' age period.

\section{Discussion}

Current research interest in nutrition is directed towards the invention of novel dietary compounds with specific effects in health promotion and reduction of the risk of disease ${ }^{(20)}$. This also holds true for specific strains of probiotics, which are associated with immune regulation, improved gut barrier function and reduction of the risk of intestinal infections and allergic diseases, thereby furnishing documentation of efficacy $^{(21)}$. Safety documentation, again, is mandatory in extending application to the general population. In the present study, we provide evidence of normal growth rates in children receiving probiotics, indicating the long-term safety of the approach. Specifically, this evidence of optimal pre- and postnatal growth encourages anticipating safe protection against metabolic diseases later in life.

The present paper is the first to report the safety aspect of the probiotic intervention starting as early as the first trimester of pregnancy. The merit of this approach lies in the possibility to evaluate the effect, if any, on pregnancy outcome. This is important in that specific strains of probiotics have been shown to contribute to the generation of $\operatorname{Th} 1, \operatorname{Th} 3$ and $\operatorname{Tr} 1$ regulatory cells ${ }^{(22,23)}$, with a counter-regulatory activity on the Th2-skewed immune responder type, with this prevailing in utero ${ }^{(24,25)}$. The normal pregnancy outcome reported here confirms no interference with the physiological equilibrium and duration of the pregnancy, concomitantly confirming the safety aspect. In view of the fact that impaired maternal glycaemia predisposes not only the mother but also the child to perinatal and later health problems ${ }^{(8)}$, the diminished risk of GDM reported in the present study may have been a contributor on reducing the adverse pregnancy outcomes.

The size of the mother and especially the diet during pregnancy influence fetal growth and development, these being mirrored in birth size ${ }^{(26,27)}$. Prepregnancy obesity, excessive weight gain during pregnancy and GDM overnourish the fetus and lead to fetal macrosomia ${ }^{(28)}$. Larger birth size, again, predisposes to later obesity ${ }^{(29,30)}$ with associated comorbidities $^{(31)}$. Previous reports from this project have already demonstrated, firstly, that dietary counselling during 
pregnancy improved the dietary intake, particularly the amount of fibre and the quality of fat intake, of the pregnant women $^{(15)}$. Secondly, we have provided clinical evidence of consistently improved plasma glucose concentrations and insulin sensitivity in healthy women during pregnancy and 12 months post partum when advantageous dietary intake is combined with probiotics ${ }^{(14)}$. The demonstration here extends these effects to fetus and neonate. Interestingly, nutrition counselling and probiotic intervention were shown in the present study to have a distinct effect on GDM; probiotics diminished the risk of the disorder, and dietary counselling during pregnancy reduced the risk of fetal overgrowth associated with it.

Considering that the maternal microbiota is a first inoculum to the development of the child's microbiota ${ }^{(32)}$, it is important to recognise that the gut microbiota has recently come to be seen as a key organ involved in host energy homoeostasis ${ }^{(9)}$, in affecting the harvest and storage of energy from the diet. Furthermore, it modulates plasma lipopolysaccharide concentrations and sets the inflammation tone to affect insulin sensitivity and thereby the risk of the metabolic syndrome ${ }^{(9,10,33,34)}$. Specific probiotic consumption may modify the intestinal microbiota composition and activity in such a way that fermentation of dietary polysaccharides is altered and gut barrier function is improved, not forgetting the capability to regulate the inflammatory pathways ${ }^{(35,36)}$. It is conceivable that the protection against GDM provided by probiotics, as described in the present report, may have been mediated via both immunomodulatory pathways and energy harvest. The diminished risk of GDM-associated fetal overgrowth, again, provided by dietary intervention may be explained by the change achieved in dietary fat composition, which also modifies the gut microbiota composition and the development of innate immunity ${ }^{(37)}$. Indeed, microbes and fatty acids are both capable of exploiting the same pathways in the innate immune system, which participates in the regulation of glucose metabolism and insulin resistance ${ }^{(38)}$.

The high incidence of GDM observed in the present study may be explained by differences in the diagnostic criteria ${ }^{(39)}$.

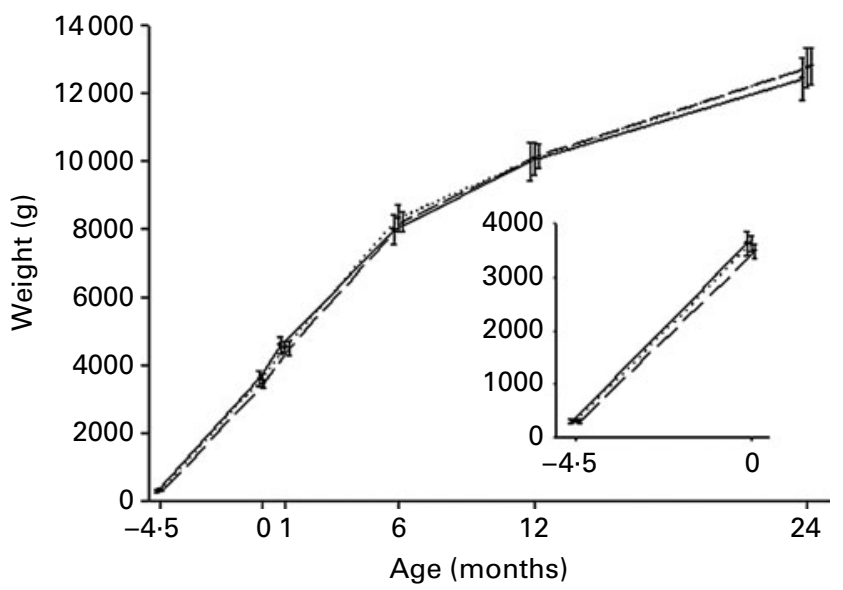

Fig. 2. Impact of probiotic-supplemented dietary intervention on perinatal growth from 20th pregnancy week to delivery and over the follow-up period to 24 months' age in diet/probiotics group ( $n$ 28), diet/placebo group ( $n$ 25) and control group $(n 17)$. Values are mean weights $(95 \% \mathrm{Cl}) . P=0.809$, ANOVA for repeated measurements, group effect. 
This notwithstanding, elevated maternal glucose levels, even within non-diabetic ranges, are of concern, because they have been shown to be related to larger offspring birth size and increased risk of interventional deliveries ${ }^{(40)}$. Hence, the findings of the present study invite acceptance of new universal guidelines for the type of threshold selection of GDM.

In view of the recent demonstration that obesity and allergic diseases share common environmental influences, i.e. a lowgrade systemic inflammation ${ }^{(41,42)}$, we have for the first time targeted a metabolically healthy at -risk population, which would most likely benefit from the dietary intervention combined with probiotics.

\section{Conclusion}

Taken together, long-term health benefits for mothers and children may be conferred by balanced maternal nutrition during pregnancy and lactation and by promoting the healthy gut microbiota in the mother and the child. The results of the present study add weight to the argument that the continuing burden of Western lifestyle diseases is modifiable. Based on the present findings, perinatal dietary counselling combined with probiotics could provide a safe and cost-effective tool in addressing the obesity epidemic.

\section{Acknowledgements}

The present study was supported by grants from the Academy of Finland, the Sigrid-Jusélius Foundation, Juho Vainio Foundation and the Social Insurance Institution of Finland. Provision of food products was by Raisio plc (Raisio, Finland), B. lactis Bb12 by Chr. Hansen (Hoersholm, Denmark) and L. rhamnosus GG by Valio Ltd (Helsinki, Finland). We would like to thank Ms Ulla-Maija Eriksson for her clinical work with the study subjects, Tuija Poussa, MSc, for statistical consultation and Robert MacGilleon, MSA, for language review of the manuscript. The authors declare that there is no personal or financial conflict of interest associated with the present paper. The authors' responsibilities were as follows: K. L. and E. I. made the study concept and design, M. N. and R. L. did the collection of the data and all authors participated in preparation of the manuscript. Conflict of interest: The authors have no conflict of interest related to the present article. The trial registration: http://www. clinicaltrials.gov/ct/gui/show/NCT00167700,section3

\section{References}

1. Lucas A (1998) Programming by early nutrition: an experimental approach. J Nutr 128, Suppl. 2, 401S-406S.

2. Sorensen HT, Sabroe S, Rothman KJ, et al. (1997) Relation between weight and length at birth and body mass index in young adulthood: cohort study. BMJ 315, 1137.

3. Parsons TJ, Power C \& Manor O (2001) Foetal and early life growth and body mass index from birth to early adulthood in 1958 British cohort: longitudinal study. BMJ 323, 1331-1335.

4. Palinski W, Yamashita T, Freigang S, et al. (2007) Developmental programming: maternal hypercholesterolemia and immunity influence susceptibility to atherosclerosis. Nutr Rev 65, 12 Pt 2, S182-S187.

5. Innis SM (2007) Human milk: maternal dietary lipids and infant development. Proc Nutr Soc 66, 397-404.
6. Howie PW, Forsyth JS, Ogston SA, et al. (1990) Protective effect of breast feeding against infection. BMJ 300, 11-16.

7. Surkan PJ, Hsieh CC, Johansson AL, et al. (2004) Reasons for increasing trends in large for gestational age births. Obstet Gynecol 104, 720-726.

8. Damm P (2009) Future risk of diabetes in mother and child after gestational diabetes mellitus. Int J Gynaecol Obstet 104, Suppl. 1, 525-526.

9. Bäckhed F, Ding H, Wang T, et al. (2004) The gut microbiota as an environmental factor that regulates fat storage. Proc Natl Acad Sci USA 101, 15718-15723.

10. Cani PD \& Delzenne NM (2007) Gut microflora as a target for energy and metabolic homeostasis. Curr Opin Clin Nutr Metab Care 10, 729-734.

11. Ley RE, Turnbaugh PJ, Klein S, et al. (2006) Microbial ecology: human gut microbes associated with obesity. Nature 444, 1022-1023.

12. Kalliomäki M, Collado MC, Salminen S, et al. (2008) Early differences in fecal microbiota composition in children predict overweight. Am J Clin Nutr 87, 534-538.

13. Kalliomäki M, Kirjavainen P, Eerola E, et al. (2001) Distinct patterns of neonatal gut microflora in infants in whom atopy was and was not developing. J Allergy Clin Immunol 107, 129-134.

14. Piirainen T, Isolauri E, Lagström $\mathrm{H}$, et al. (2006) Impact of dietary counselling on nutrient intake during pregnancy: a prospective cohort study. Br J Nutr 96, 1095-1104.

15. Laitinen K, Poussa T \& Isolauri E (2009) Probiotics and dietary counselling contribute to glucose regulation during and after pregnancy: a randomised controlled trial. Br J Nutr 101, 1679-1687.

16. Aaltonen J, Ojala T, Laitinen K, et al. (2008) Evidence of infant blood pressure programming by maternal nutrition during pregnancy: a prospective randomized controlled intervention study. J Pediatr 152, 79-84.

17. Becker W, Lyhne N, Pedersen AN, et al. (2004) Nordic nutrition recommendations 2004 - integrating nutrition and physical activity. Scand J Nutr 48, 178-187.

18. Nordic Working Group on Diet and Nutrition (1996) Nordic nutrition recommendations. Scand J Nutr 40, 161-165.

19. Metzger BE \& Coustan DR (1998) Summary and recommendations of the fourth international workshop-conference on diabetes mellitus. The organizing committee. Diabetes Care 21, Suppl. 2, B161-B167.

20. Schwager J, Mohajeri MH, Fowler A, et al. (2008) Challenges in discovering bioactives for the food industry. Curr Opin Biotechnol 19, 66-72.

21. Douglas LC \& Sanders ME (2008) Probiotics and prebiotics in dietetics practice. J Am Diet Assoc 108, 510-521.

22. He F, Morita H, Ouwehand AC, et al. (2002) Stimulation of the secretion of pro-inflammatory cytokines by Bifidobacterium strains. Microbial Immunol 46, 781-785.

23. Christensen HR, Frøkiær H \& Pestka JJ (2002) Lactobacilli differentially modulate expression of cytokines and maturation surface markers in murine dendritic cells. J Immunol 168, 171-178.

24. Piccini MP, Beloni L, Livi C, et al. (1998) Defective production of both leukemia inhibitory factor and type 2 T-helper cytokines by decidual T cells in unexplained recurrent abortions. Nat Med 4, 1020-1024.

25. Prescott SL, Macauba C, Smallacombe T, et al. (1999) Development of allergen-specific T-cell memory in atopic and normal children. Lancet 353, 196-200.

26. Godfrey K, Robinson S, Barker DJ, et al. (1996) Maternal nutrition in early and late pregnancy in relation to placental and foetal growth. BMJ 312, 410-414.

27. Barker DJ (2004) The developmental origins of adult disease. J Am Coll Nutr 23, Suppl. 6, 588S-595S. 
28. Catalano P (2002) The diabetogenic state of maternal metabolism in pregnancy. NeoReviews 3, e165-e171.

29. Baird J, Fisher D, Lucas P, et al. (2005) Being big or growing fast: systematic review of size and growth in infancy and later obesity. BMJ 331, 929-934.

30. Druet C \& Ong KK (2008) Early childhood predictors of adult body composition. Best Pract Res Clin Endocrinol Metab 22, 489-502.

31. Boney CM, Verma A, Tucker R, et al. (2005) Metabolic syndrome in childhood: association with birth weight, maternal obesity, and gestational diabetes mellitus. Pediatrics 115, e290-e296.

32. Mackie RI, Sghir A \& Gaskins HR (1999) Developmental microbial ecology of the neonatal gastrointestinal tract. Am J Clin Nutr 69, 1035S-1045S.

33. Turnbaugh PJ, Ley RE, Mahovald MA, et al. (2006) An obesityassociated gut microbiome with increased capacity for energy harvest. Nature 444, 1027-1031.

34. Cani PD, Amar J, Iglesias MA, et al. (2007) Metabolic endotoxemia initiates obesity and insulin resistance. Diabetes 56, 1761-1772.

35. Isolauri E (1999) Probiotics and gut inflammation. Curr Opin Gastroenterol 15, 534-537.
36. Cani PD, Possemiers S, Van de Wiele T, et al. (2009) Changes in gut microbiota control inflammation in obese mice through a mechanism involving GLP-2-driven improvement of gut permeability. Gut 58, 1091-1103.

37. Calder PC (2006) Polyunsaturated fatty acids and inflammation. Prostaglandins Leukot Essent Fatty Acids 75, 197-202.

38. Shi H, Kokoeva MV, Inouye K, et al. (2006) TLR4 links innate immunity and fatty acid induced insulin resistance. J Clin Invest 116, 3015-3025.

39. Yogev Y, Metzger BE \& Hod M (2009) Establishing diagnosis of gestational diabetes mellitus: Impact of the hyperglycemia and adverse pregnancy outcome study. Semin Foetal Neonatal Med 14, 94-100.

40. HAPO Study Cooperative Research Group, Metzger BE, Lowe LP, et al. (2008) Hyperglycemia and adverse pregnancy outcomes. N Engl J Med 358, 1991-2002.

41. Weiss ST (2005) Obesity: insight into the origins of asthma. Nat Immunol 6, 537-539.

42. Shore SA (2008) Obesity and asthma: possible mechanism. J Allergy Clin Immunol 121, 1087-1093. 